\title{
Functional Critical Aortic Stenosis with Transient Retrograde Flow in a Neonate with Left Diaphragmatic Hernia
}

\author{
Daisuke Kobayashi, MD, Michael D. Pettersen, MD, and Sanjeev Aggarwal, MD \\ Division of Cardiology, Carman and Ann Adams Department of Pediatrics, Children's Hospital of Michigan, Wayne State \\ University School of Medicine, Detroit, Mich, USA
}

\begin{abstract}
A B S T R A C T
We report a neonate with left congenital diaphragmatic hernia and severe left ventricular dysfunction, in whom the blood flow in the transverse arch and its branches was supported in a retrograde fashion by patent ductus arteriosus. There was only minimal antegrade flow across the aortic valve and hemodynamic physiology resembled critical aortic stenosis, necessitating the immediate use of prostaglandin E1 infusion to maintain the patent ductus arteriosus.
\end{abstract}

Key Words. Diaphragmatic Hernia; Patent Ductus Arteriosus; Retrograde Arch Flow; Prostaglandin E1; Patent Ductus Arteriosus

\section{Introduction}

$\mathrm{R}$ etrograde flow in the ascending and transverse aorta predicts survival in infants with critical aortic valve stenosis (AS) after biventricular repair. ${ }^{1}$ Critical AS is a ductal-dependent lesion requiring use of prostaglandin $\mathrm{E} 1$ (PGE) to maintain systemic circulation with blood supply from patent ductus arteriosus (PDA). ${ }^{2}$ Infants with congenital diaphragmatic hernia $(\mathrm{CDH})$ have been reported to have impaired left ventricular (LV) function. ${ }^{3}$ Thankavel et al. reported a neonate with left $\mathrm{CDH}$ in whom a retrograde flow in the transverse aortic arch was present in the setting of normal LV function. ${ }^{4}$ We present a neonate with left CDH with severe LV dysfunction, a retrograde flow in the transverse arch, and minimal antergrade flow across the aortic valve. The hemodynamics in our case resembled critical aortic stenosis.

\section{Case Report}

A female infant with prenatal diagnosis of $\mathrm{CDH}$ at 20 weeks gestation was born at 38 weeks gestation via normal vaginal delivery to a 29-year-old G4P3 mother. Prenatal history was unremarkable except for the diagnosis of CDH and maternal use of tobacco. Chromosomal testing of amniotic fluid showed a normal female katyotype (46XX). Her birth weight was $2.9 \mathrm{~kg}$ with Apgar scores of 8 and

(C) 2012 Wiley Periodicals, Inc.
9 at 1 and 5 minutes, respectively. Immediately after birth, she was intubated and umbilical arterial and venous lines were placed. Capillary refill was prolonged at 4-5 seconds with cold extremities and diminished rapid pulses. Blood pressure was $57 / 35 \mathrm{~mm} \mathrm{Hg}$ with a mean of $44 \mathrm{~mm} \mathrm{Hg}$. Heart rate was 165 beats per minute. Her oxygen saturation was $70 \%$ on $\mathrm{FiO} 2100 \%$ (on ventilator). Breath sounds were reduced on left side and heart sounds were audible on the right side of the chest. The first and second heart sounds were normal without appreciable murmurs, click, or gallop. Abdomen was flat. These findings were consistent with left $\mathrm{CDH}$. Her initial arterial blood gas showed severe respiratory acidosis with $\mathrm{pH}$ 6.99, $\mathrm{pCO}_{2} 74 \mathrm{~mm} \mathrm{Hg}, \mathrm{pO}_{2} 43 \mathrm{~mm} \mathrm{Hg}$, base excess $-13 \mathrm{mmol} / \mathrm{L}$. Initial echocardiography showed normal four chamber intracardiac anatomy with normal LV size (LV internal dimension in diastole $21 \mathrm{~mm}, z$-score +1.65$)$. Mitral valve annulus measured $9.1 \mathrm{~mm}(z$-score -0.1$)$. Aortic valve annulus measured $5.5 \mathrm{~mm}(z$-score +0.36$)$. There was normal left aortic arch without evidence of coarctation of the aorta. A large PDA was seen with a large right-to-left shunt. Moderate left-to-right shunt was seen through secundum atrial septal defect. Pulmonary venous return was normal. The LV function was severely reduced (ejection fraction $18 \%)$. The calculated cardiac output was $0.13 \mathrm{~L} / \mathrm{min}$ with velocity time integral at the aortic

Congenit Heart Dis. 2013;8:E85-E87 


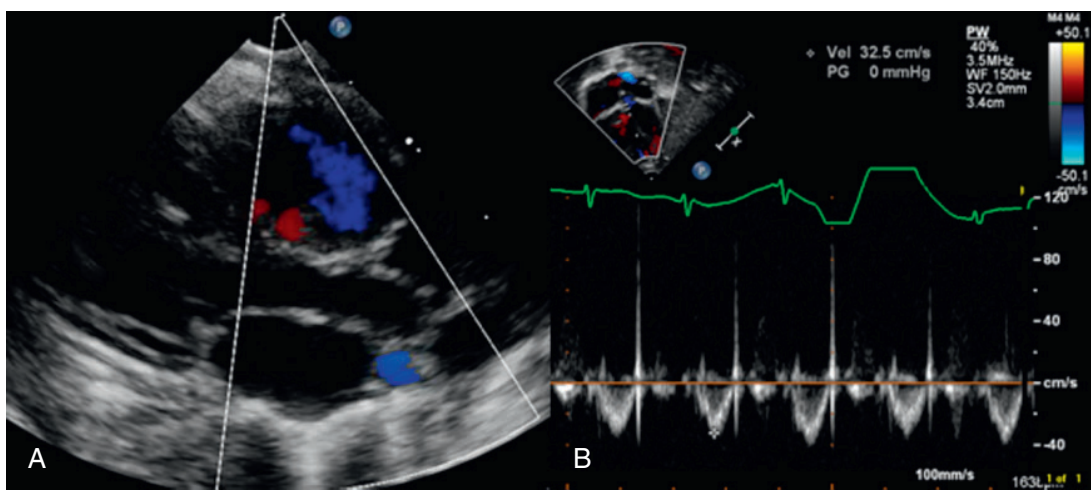

Figure 1. Echocardiography. (A) Parasternal long-axis view, showing the widely opened aortic valve. Color flow Doppler showed no evidence of aortic stenosis. (B) Pulsed-wave Doppler interrogation at the aortic valve in the apical view. Minimal antegrade flow is noted across the aortic valve, with peak velocity of $0.3 \mathrm{~m} / \mathrm{s}$ and velocity time integral of $3.5 \mathrm{~cm}$.

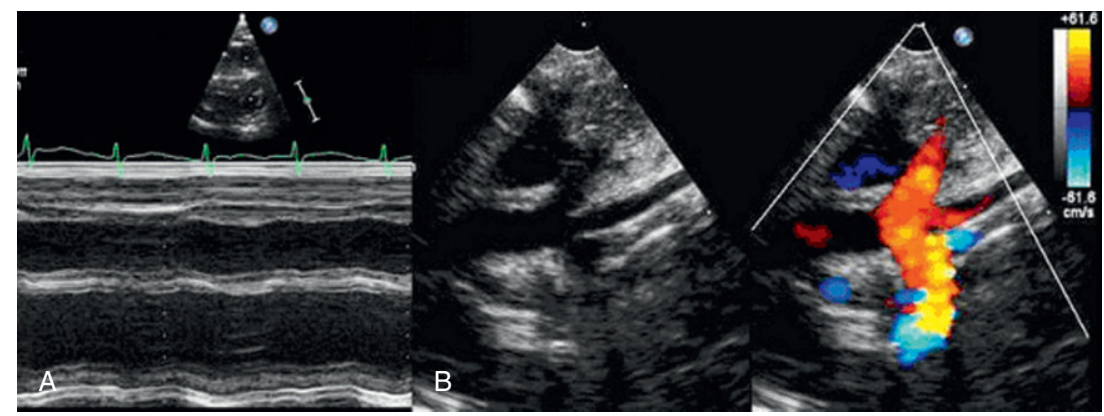

Figure 2. Echocardiography. (A) M-mode in short-axis view at the level of papillary muscles, showing severely decreased left ventricular function with a ejection fraction of $18 \%$. (B) Suprasternal long axis view with color Doppler interrogation, demonstrating the retrograde flow in the transverse aortic arch.

valve of $3.5 \mathrm{~cm}$ (Figure 1). The myocardial performance index of LV was elevated at 0.79 . Retrograde flow was observed in the transverse aortic arch, with minimal antergrade flow across the normal aortic valve (Figure 2). There was mild mitral regurgitation and moderate tricuspid regurgitation with estimated systolic right ventricular pressure of $55 \mathrm{~mm} \mathrm{Hg}$ (near systemic). Blood pressures in the right arm and leg were 57/42 (mean 47) and 61/36 $\mathrm{mm} \mathrm{Hg}$ (mean 45), respectively. To maintain the systemic cardiac output, PGE infusion was immediately started at $0.05 \mathrm{mCg} / \mathrm{kg} / \mathrm{min}$ to keep the PDA open. She also required inotropic support to maintain the blood pressures. Head ultrasound showed no evidence of intracranial hemorrhage or arteriovenous malformation. Her respiratory acidosis improved transiently with administration of nitric oxide $(20 \mathrm{ppm})$ and high frequency oscillator ventilation. However, she became profoundly hypoxic $\left(\mathrm{PaO}_{2} 8 \mathrm{~mm} \mathrm{Hg}\right)$ with persistent metabolic acidosis ( $\mathrm{pH} 7.16$ and base excess $-11 \mathrm{~mm} \mathrm{Hg}$ ). After extensive discussion with her family about her critical condition and grim prognosis, extracorporeal membrane oxygenation was not pursued. She expired at the age of 24 hours.

\section{Discussion}

We report a neonate with left $\mathrm{CDH}$, severe $\mathrm{LV}$ dysfunction and retrograde flow in the transverse aortic arch. The hemodyanmic in our case resembled critical AS and necessitated the immediate use of PGE.

Fetuses with left $\mathrm{CDH}$ have been reported to have mild to moderate left heart hypoplasia and reduced flow through aortic valve. ${ }^{5}$ The postnatal echocardiography in 65 neonates with left $\mathrm{CDH}$ showed smaller left sided structure with a mean $z$-score of LV end-diastolic volume $-1.10 \pm 1.08$, mitral valve diameter $-0.91 \pm 1.10$, and aortic valve diameter $-0.62 \pm 1.04 .^{5}$ Infants with $\mathrm{CDH}$ have significantly decreased LV function and LV 
dysfunction is associated with death and adverse outcomes. $^{3}$

Thankavel et al. reported a neonate with left $\mathrm{CDH}$ and a retrograde flow in the transverse arch. ${ }^{4}$ The right-to-left shunt across the PDA directed the blood flow into the transverse arch in a retrograde fashion. In this case, there was a suspicion of coarctation of aorta with a $z$-score of aortic isthmus -2.64 . Therefore, the PDA appears to play a role in acting as a pop-off of pulmonary hypertension as well as maintaining the blood flow in the descending aorta. In contrast, in our case, LV function was severely decreased with minimal antegrade flow across the aortic valve. There was no evidence of coarctation of aorta. The direction of shunt was left to right at the atrial level. The hemodyanmics in our case resembles functional critical AS and the blood flow derived from the PDA was the sole source of systemic circulation. The PGE was critical to keep the PDA patent in order to maintain systemic cardiac output.

Similar to the role of PGE in dysfunctional $\mathrm{LV}$, the use of PGE is life saving in symptomatic neonatal Ebstein's anomaly. ${ }^{6,7}$ Dysfunctional and hypoplastic right ventricle and severe tricuspid regurgitation can cause functional pulmonary valve atresia and ductal dependent pulmonary circulation.

The antegrade flow in the ascending and transverse aorta was a strong predictor for survival after biventricular repair in infants with critical AS. ${ }^{1}$ In the study of 28 infants with critical AS, retrograde flow in the ascending and transverse aorta was observed in 9/25 (36\%) and 13/28 (46\%). ${ }^{1}$ The LV function was depressed in all patients (mean ejection fraction $16-27 \%$ ).

The effect of CDH on coronary blood flow has not been investigated. Harada et al. reported an infant with critical AS in whom the coronary blood flow was measured by Doppler echocardiography before and after balloon valvuloplasty. ${ }^{8}$ The coronary flow volume increased after the procedure, indicating that coronary blood flow appeared impaired even with a widely open PDA providing retrograde flow. In our case, coronary blood flow was likely to be impaired by both severe LV dysfunction and resultant retrograde aortic flow.

\section{Author Contribution}

DK wrote the manuscript and collected the data for review of literature. MDP and SA critically revised and approved it.
Corresponding Author: Daisuke Kobayashi, MD, Division of Cardiology, Carman and Ann Adams Department of Pediatrics, Children's Hospital of Michigan, 3901 Beaubien Blvd, Detroit, MI 482012119, USA. Tel: (+1) 313745-5481; Fax: (+1) 313-9930894; E-mail: dkobayas@dmc.org

Conflict of interest: No conflict of interest, grants, or other financial support.

Accepted in final form: April 3, 2012.

\section{References}

1 Kovalchin JP, Brook MM, Rosenthal GL, Suda K, Hoffman JI, Silverman NH. Echocardiographic hemodynamic and morphometric predictors of survival after two-ventricle repair in infants with critical aortic stenosis. 7 Am Coll Cardiol. 1998;32: 237-244.

2 Artman M, Boucek RJ Jr, Hammon J, Graham TP Jr. Emergency palliation of critical valvular aortic stenosis. A new application of prostaglandin E1. Am 7 Dis Child. 1983;137:339-340.

3 Aggarwal S, Stockmann P, Klein MD, Natarajan G. Echocardiographic measures of ventricular function and pulmonary artery size: prognostic markers of congenital diaphragmatic hernia? I Perinatol. 2011; 31:561-566.

4 Thankavel PP, Ramaciotti C, Lemler MS. A neonate with left congenital diaphragmatic hernia and transient retrograde flow in the aortic arch. Pediatr Cardiol. 2011;32:1207-1208.

5 Vogel M, McElhinney DB, Marcus E, Morash D, Jennings RW, Tworetzky W. and Significance outcome of left heart hypoplasia in fetal congenital diaphragmatic hernia. Ultrasound Obstet Gynecol. 2010;35:310-317.

6 Gaio G, Santoro G, Esposito R, et al. Patent ductus arteriosus "stenting" as a life-saving approach in severe neonatal Ebstein's anomaly. 7 Cardiovasc Med (Hagerstown). 2007;8:937-939.

7 Santoro G, Palladino MT, Russo MG, Calabrò R. Neonatal patent ductus arteriosus recanalization and stenting in critical Ebstein's anomaly. Pediatr Cardiol. 2008;29:176-179.

8 Harada K, Toyono M, Tamura M. Effects of balloon valvuloplasty on left anterior descending coronary artery blood flow in a neonate with critical aortic stenosis with transthoracic doppler echocardiography. 7 Am Soc Echocardiogr. 2003;16: 88-90. 